\title{
Evolución de la expansión urbana y riesgos para la agricultura de proximidad en el sur de Córdoba, Argentina
}

\begin{abstract}
Emiliano Cahe. Universidad Nacional de Río Cuarto, Córdoba, Argentina. Jorge de Prada. Universidad Nacional de Río Cuarto, Córdoba, Argentina.
\end{abstract}

RESUMEN | Los efectos indeseados de la dispersión urbana en el territorio han sido estudiados en diferentes escalas, aunque son escasos los estudios que comparan su dinámica con otras formas de poblamiento. El artículo cuantifica la expansión urbana y riesgos para la agricultura de proximidad, y la compara con formas alternativas de poblamiento. Mediante Quantum-Gis, imágenes históricas de Google Earth y la relación perímetro real-teórico del área urbana, se identificó el poblamiento de 69 localidades del sur de Córdoba, Argentina. Los resultados muestran dispersión urbana creciente: la tasa anual promedio de crecimiento poblacional fue $1,2 \%$, mientras que la tasa del área urbana pasó de 3,4\% a 4,1\% entre los períodos 2001-2010 y 2010 2018, respectivamente. Se redujo $88 \%$ la provisión de alimentos de proximidad, y al ańo 2040, se triplicaría el área urbana respecto de poblamientos más compactos. Existe una brecha para minimizar la conversión de tierras rurales y potenciar la agricultura de proximidad.

PALABRAS CLAVE | periferia urbana, planificación regional, relación campo-ciudad.

ABSTRACT | The unwanted effects of urban dispersion in the territory have been described at different scales, although there are few studies that compare its dynamics with other forms of settlement. The article quantifies urban expansion and risks to local agriculture, and compares them with alternative forms of settlement. Using Quantum-Gis, historical images from Google Earth and the real-theoretical perimeter relationship of the urban area, the settlement of 69 towns in the south of Córdoba, Argentina was identified. The results show increasing urban dispersion, the average annual population growth rate was $1.2 \%$ while the rate of the urban area went from $3.4 \%$ to $4.1 \%$ between periods 2001 2010 and 2010-2018 respectively. Provision of local food was reduced by 88\%, and by 2040 , the urban area would triple with respect to more compact settlements. There is a gap to minimize the conversion of rural land and promote local agriculture.

KEYWORDS | urban periphery, regional planning, countryside-city relationship. 


\section{Introducción}

El fenómeno de expansión urbana y el desarrollo sostenible de una región son procesos, a menudo, unos a otros excluyentes. La franja urbano-rural constituye el territorio en el cual la expansión urbana se manifiesta con transformaciones y efectos directos para la sostenibilidad. Este territorio es una zona de transición entre lo urbano y lo rural, con múltiples funciones y servicios que complementan el desarrollo de estos espacios y contribuyen idealmente a él (Gallent, 2006; Scott et al., 2013). Sin embargo, cuando algunas funciones de este territorio, como la reserva de espacio físico para poblamientos urbanos futuros y la producción de alimentos de proximidad, no se complementan sinérgicamente a otras funciones de este espacio y el poblamiento urbano es disperso, se corre el riesgo de insostenibilidad (Gallent \& Shaw, 2007; Kasanko et al., 2006; Lopez, 2014).

Los efectos no deseados y riesgos del patrón de urbanización disperso son varios. En la dimensión ambiental, la expansión urbana dispersa ha aumentado: i) las emisiones de gases de efecto invernadero a nivel global (Dodman, 2009); ii) la fragmentación del paisaje en diversas regiones (Alberti, 2005; Pauleit et al., 2005); iii) el consumo de energía fósil; iv) la conversión de tierras agrícolas y humedales a zonas urbanas en diferentes estados y países de los Estados Unidos y Europa, respectivamente (Alberti, 2005; Hasse \& Lathrop, 2003; Munton, 2009); v) la presencia de eventos de calor extremo en algunas áreas metropolitanas de los Estados Unidos (Stone et al., 2010); vi) el desequilibrio hidrológico de las cuencas al suroeste de Alemania (Samaniego \& Bárdossy, 2006); vii) el riesgo de inundaciones, ilustrado con casos de El Salvador y la Argentina (Bertoni, 2006); y viii) las ineficiencias en la gestión de residuos y efluentes urbanos en ciudades de Argentina (López Goyburu, 2016). En la dimensión social, la expansión urbana dispersa ha generado, en diferentes localidades de la región pampeana de Argentina, mayores conflictos urbanos rurales por exposición y riesgo de la población a contaminación por agroquímicos (Cabrini et al., 2018; Galfioni et al., 2013), y también ha acrecentado la brecha entre población más y menos vulnerable en ciudades de Argentina (Río Cuarto, Córdoba) y España (Cataluña) (Galfioni et al., 2013; Puig, 2016). En tanto, en la dimensión económica, la dispersión urbana ha incrementado los costos de equipamiento urbano, infraestructura y cobertura de servicios públicos en diferentes ciudades de Estados Unidos y España (Carruthers \& Ulfarsson, 2003; Hortas-Rico, 2014), como también los costos de producción de alimentos de proximidad en la ciudad de Córdoba, Argentina (Boccolini \& Giobellina, 2018).

No obstante, existen al menos seis patrones de urbanización más sostenibles. El primer patrón, ciudad inteligente, usa tecnologías de información y comunicación en diferentes ámbitos, tales como seguridad, salud y movilidad urbana, para lograr efectos deseados referidos a la reducción del consumo de energía fósil y las emisiones de gases de efecto invernadero (Neirotti et al., 2014). El segundo patrón, ciudad verde, se caracteriza por el desarrollo de infraestructuras urbanas que usan la vegetación para mitigar los efectos del cambio climático y para la ornamentación (Moore, 2016). El tercer patrón, formas urbanas compactas, minimiza el uso del suelo urbano (Burton et al., 2003; Jabareen, 2006; Jenks \& Dempsey, 2005). El 
cuarto patrón, ciudad neotradicional, representa una nueva forma de urbanismo con criterios de uso mixto del suelo, densidad, compacidad, movilidad sostenible y diseño de espacios verdes (Jabareen, 2006). El quinto patrón, ciudad contenida, es similar al patrón anterior y no enfatiza en los espacios verdes (Jabareen, 2006). El sexto patrón, ecociudad, reúne características del patrón formas urbanas compactas y ciudad inteligente, en aspectos como movilidad urbana, construcciones resilientes y uso pasivo de energía, entre otros (Jabareen, 2006; Gaffron et al., 2008; Liao \& Chern, 2015).

Aunque estos patrones comparten similitudes en sus definiciones, algunos presentan mejores características para el poblamiento urbano sostenible. De hecho, Jabareen (2006) valora cuatro de los seis patrones descriptos con siete criterios. Los criterios son densidad, diversidad, uso mixto del suelo, compacidad, movilidad sostenible, diseño solar pasivo y diseños ecológicos-verdes. Los patrones compacto y ecociudad son mejor valorados en los criterios de diversidad, uso mixto del suelo, densidad, compacidad y movilidad sostenible.

En Argentina, varios estudios describen las implicancias de la expansión urbana dispersa. Este fenómeno ha sido estudiado en el gran Buenos Aires (Barsky, 2005) y en las localidades de Mar del Plata (Zulaica \& Ferraro, 2013), de Bahía Blanca (Sereno \& Serer Santarelli, 2012; Urriza \& Garriz, 2014), de Santa Fe (Venier, 2012), de Córdoba (Boccolini \& Giobellina, 2018), de Río Cuarto (de Prada et al., 2017b; Maldonado \& Campanella, 2004; Montero, 2011), de Santa Eufemia (de Prada et al., 2017a) y en la provincia de Córdoba (Margonari \& Menendez, 2019). En Río Cuarto y Santa Eufemia, la expansión urbana dispersa fue proyectada y comparada con otras formas de urbanización más sostenibles. En su mayoría estos trabajos corresponden a estudios de casos donde la presencia del patrón urbano disperso se describe con escasa precisión, a excepción del trabajo de Margonari y Menendez (2019). Estos autores elaboraron índices a partir de imágenes de satélites 2017-2018 (siguiendo la metodología de Angel et al., 2012) para cuantificar la dispersión urbana en la provincia de Córdoba, denominada "periurbanización". Sus resultados alertan sobre la urbanización dispersa y queda el interrogante, especialmente para el sur de Córdoba, de si la dinámica de este fenómeno es creciente o no. También está pendiente determinar cuáles pueden ser sus implicancias, comparadas con otros patrones de urbanización más sostenibles en cuanto a costo de la tierra, abastecimiento de alimentos relevantes para la seguridad alimentaria, la relación de contacto entre el territorio urbano y el rural. Este trabajo intenta cerrar estas brechas de conocimiento.

El objetivo del artículo es cuantificar la evolución de la expansión urbana entre los ańos 2001 y 2018 y comparar la tendencia de poblamiento con dos formas urbanas alternativas al año 2040, considerando los riesgos para la agricultura de proximidad y la conversión de tierras en la franja urbana rural de 69 localidades de la región sur de Córdoba, Argentina.

La comparación entre patrones de poblamiento se realiza con parámetros tomados de la bibliografía. La evolución de la expansión urbana permite visualizar si las localidades con expansión urbana dispersa crecen o, simplemente, son localidades aisladas con un comportamiento específico. En tanto, la prognosis, según 
lo sugerido por Ackoff (1994), nos permite precisar la problemática existente y alertar sobre la necesidad de cambios en la relación población-actividades (Gómez Orea, 2008) y el sistema territorial. En este sentido, los patrones de urbanización alternativos nos ayudan a dimensionar las ventajas de los posibles cambios.

Las principales contribuciones de este artículo son, una metodológica y dos empíricas. Se presenta una métrica del paisaje simple basada en relaciones perímetro-área para identificar la dispersión urbana, donde 'paisaje' refiere a la estructura física (uso-cobertura del suelo) de la forma urbana (Kasanko et al., 2006; Schwarz, 2010). En segundo lugar, se generan datos primarios sobre la dinámica de expansión de las manchas urbanas en 69 localidades del sur de la provincia de Córdoba. En tercer lugar, se cuantifican las implicancias no deseadas, potencialmente evitables, si se adoptan patrones urbanos más compactos.

Tras esta introducción, el trabajo se organiza de la siguiente forma. Se describe la metodología general y los parámetros usados; luego se muestran los resultados alcanzados y la discusión de los mismos. Por último, se compara la tendencia de poblamiento con otras formas alternativas, y se arriba a una conclusión.

\section{Metodología}

\section{Área de estudio}

El área de estudio es el sur de la provincia de Córdoba, Argentina $\left(48.183 \mathrm{~km}^{2}\right)$ y se integra por 69 localidades que se distribuyen entre los departamentos de Río Cuarto, Juárez Celman, Roque Sáenz Peña y General Roca (Figura 1).

Figura I | Área de estudio. Sur de Córdoba, Argentina
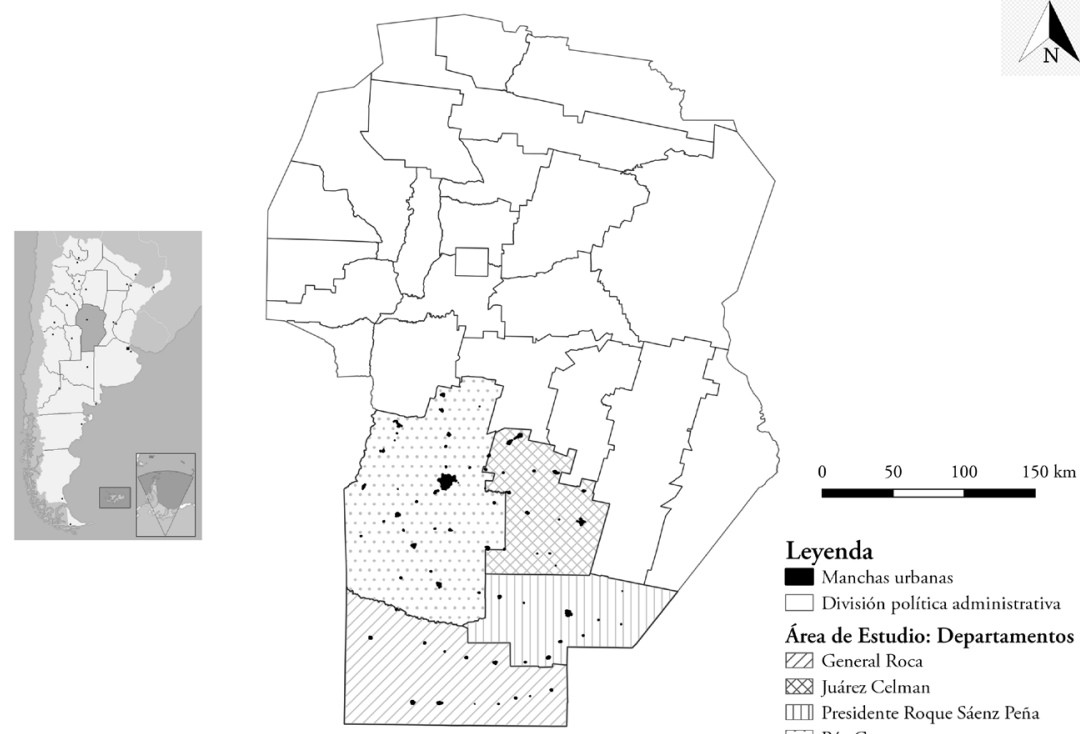

Leyenda

Manchas urbanas

$\square$ División política administrativa

Área de Estudio: Departamentos

$\square \triangle$ General Roca

XXX Juárez Celman

Iㅔ Presidente Roque Sáenz Peña

$\therefore$ Río Cuarto

FUENTE: ELABORACIÓN PROPIA 


\section{Análisis espacial de la expansión urbana}

La evolución de la expansión urbana se analizó durante dos períodos, 2001-2010 y 2010-2018. En tanto, la prognosis de expansión urbana y los patrones de poblamiento proyectados se consideran al año 2040.

Se elaboraron cinco indicadores: mancha urbana, área de riesgo de la franja urbano-rural, área afectada por expansión urbana, todas medidas en hectáreas; perímetro de la mancha urbana y pérdida de renta agrícola (PRA), medidos en kilómetros y millones de pesos constantes por año (\$c Mм año-1), respectivamente. La mancha urbana representa el área urbanizada de cada localidad y de los barrios residenciales (abiertos, cerrados, aislados) adyacentes a la misma. El área de riesgo de la franja urbano-rural se refiere a los espacios con diversos usos emergentes por precios de la tierra y oportunidades del mercado inmobiliario (residencias rurales; y áreas productivas, recreativas, industriales o en especulación), las cuales pueden estar en conflicto si no existen zonas de amortiguación o espacios complementarios entre usos incompatibles (p.e., un barrio residencial en el medio de actividades agrícolas extensivas). El área afectada por la expansión urbana es la sumatoria de las variables 'mancha urbana' y 'área de riesgo' de la franja urbano-rural. En tanto, el perímetro de la mancha urbana representa el límite entre dos territorios contrastantes, lo urbano y lo rural.

FIGURA 2 | Evolución de las manchas urbanas en el área de estudio, años 20012018. Caso aumentado: localidades del Gran Río Cuarto, Córdoba

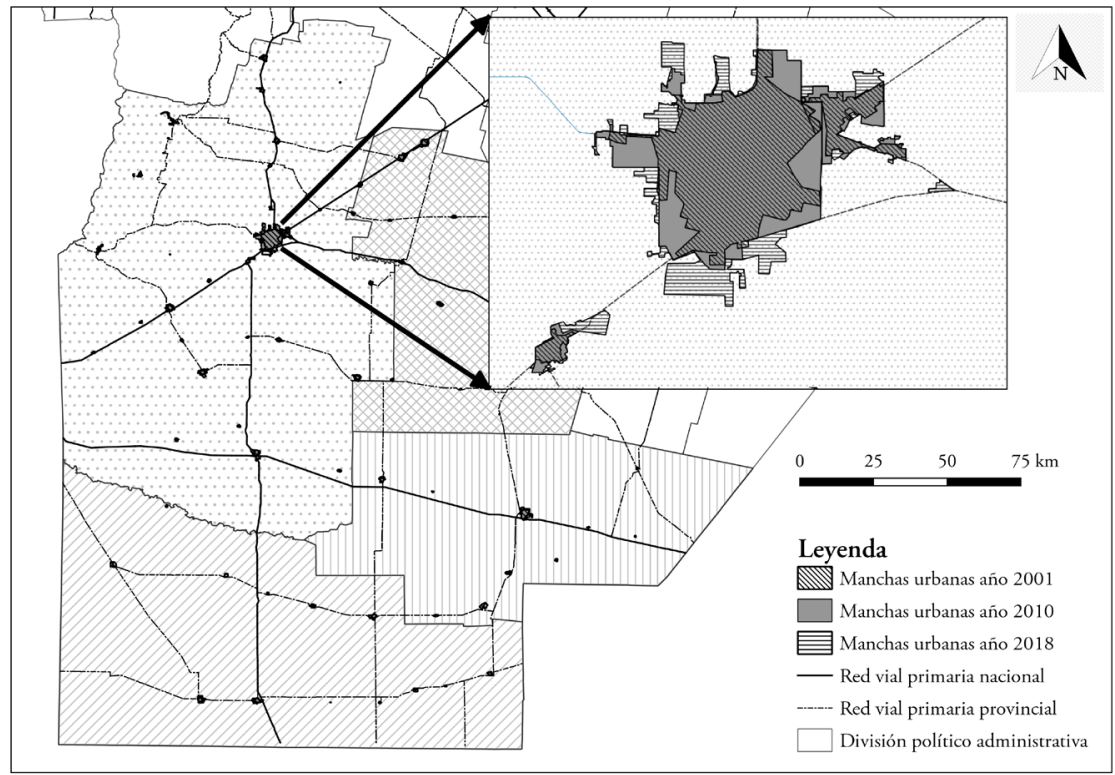

FUENTE: ELABORACIÓN PROPIA 
Los datos de población urbana (años 2001 y 2010) se tomaron de censos nacionales (Instituto Nacional de Estadística y Censos [InDEC], 2001, 2010), y mediante un modelo geométrico (Torres-Degró, 2017) se estimaron los años 2018 y 2040.

Se utilizó Quantum Gis e imágenes históricas de Google Earth para identificar la expansión urbana en las 69 localidades y elaborar los indicadores. En primer lugar, se importó al sistema de información geográfico (SIG) la capa "urbanizaciones_2010", extraída del proyecto Bases ambientales para el ordenamiento territorial del espacio rural de la provincia de Córdoba (BAOTCba, 2010). En segundo lugar, para los años 2001 y 2018, se digitalizaron las manchas urbanas de cada localidad siguiendo el uso residencial del suelo (Figura 2). En tercer lugar, se digitalizó el área de riesgo de la franja urbano-rural (Figura 3). Esta última se elaboró como la diferencia entre la capa mancha urbana y una envolvente. La envolvente es una capa de polígonos creada con la función envolvente convexa de Quantum Gis, la cual une los puntos extremos de las manchas urbanas. La diferencia entre capas (mancha urbana-envolvente) se realizó con la función diferencia simétrica de Quantum Gis.

\section{Identificación del patrón de urbanización}

Diferentes métodos son usados para precisar el patrón de la expansión urbana; sin embargo, los más difundidos se asocian con métricas de la mancha urbana y datos de población (Schwarz, 2010). Esto permite generar índices e indicadores para diferenciar entre patrones de poblamiento. Por ejemplo, Kasanko et al. (2006) clasificaron las ciudades europeas en dispersas y compactas a partir de cinco indicadores, compuestos con datos del paisaje y de población. Schneider y Woodcock (2008) usaron la extensión del área urbana impermeabilizada como indicador para clasificar poblamientos urbanos en compactos, dispersos, fragmentados y extensivos en 25 ciudades medianas y con diferente desarrollo económico de Asia, América, Europa y África. En tanto, Gao et al. (2016) determinaron con datos de población y métricas de la mancha urbana un índice de expansión urbana dispersa (IEUD) para diferentes ciudades de China. El IEUd se elabora como la diferencia entre la tasa de crecimiento del área de la mancha urbana y la tasa de crecimiento de población urbana, donde un IEUD mayor a cero indica poblamiento disperso, y viceversa. Este índice tiene como ventaja que es simple en su forma de cálculo y fácil de comunicar, pero no captura el incremento de las zonas de contacto entre el territorio urbano y el rural. En este trabajo se clasifican los patrones de poblamiento con el IEUD de acuerdo con Gao et al. (2016), y se complementa con otro indicador basado en la relación entre el perímetro real y teórico de la mancha urbana, Ratio PMU, que pone de manifiesto la zona de contacto urbano-rural y clasifica al poblamiento urbano como disperso y no disperso, de acuerdo con la siguiente expresión:

$$
\begin{aligned}
& \text { Ratio } P M U_{i t}=\frac{P M U R_{i t}}{P M U T_{i t}} \leq \propto \quad \text { el poblamiento urbano es No disperso; } \\
& \text { de otro modo (Ratio } P M U_{i t}>\alpha \text { ) el poblamiento urbano es Disperso. }
\end{aligned}
$$

donde PMUT representa el perímetro teórico de la mancha urbana equivalente a la figura geométrica que mejor represente el sistema de poblamiento urbano de la 
localidad; y PMUR es el perímetro real de la mancha urbana; los subíndices $i$ representan las localidades (de 1, 2, ., 69) y $t$ representa el tiempo (2001, 2010 y 2018). El PMUT se considera como la figura geométrica de un cuadrado, por ser la forma más representativa en las localidades del sur de Córdoba, en general asociadas a una plaza central y vías férreas.

Para discriminar entre poblamientos es necesario precisar un umbral de corte, $\alpha$. Se utilizó un umbral de corte $\alpha=1,6$ y se realizó un análisis de sensibilidad al umbral entre valores 1,4 - 1,8 para ver si la cantidad de localidades con poblamiento disperso y no disperso cambia significativamente. Por último, de acuerdo con Huang et al. (2007), se evaluó en las 69 formas urbanas el índice de compacidad del parche más grande (CLIP, en este trabajo indicado como IC) para visualizar, entre periodos, si las formas urbanas dispersas y no dispersas identificadas presentaban valores bajos y altos del índice, respectivamente.

La prognosis 2040 de los indicadores elaborados se realizó de acuerdo con el patrón de crecimiento detectado (lineal o geométrico). El valor de prognosis de cada indicador se realiza por localidad, y luego según la sumatoria de los 69 casos bajo estudio (Tabla 3). Para la población adicional estimada (2018-2040), se contrasta la prognosis de poblamiento urbano con patrones compactos de urbanización, en estrategias de agrupamiento (Burton, 2000; Jenks \& Dempsey, 2005), con dos variantes de densidad de población: Compacto-1 (25 hab. ha $\left.{ }^{-1}\right)$ y Compacto-2 (75 hab. ha ${ }^{-1}$ ).

\section{Expansión urbana y renta agraria}

Una de las principales transformaciones de la expansión urbana dispersa es la conversión de tierras rurales a urbanas a un ritmo mayor a lo deseado y, consecuentemente, la pérdida de usos agrarios por los conflictos generados. Este riesgo se valora mediante el análisis beneficios-costos, a través de la pérdida de renta económica de las tierras agrarias que son afectadas a usos urbanos. Para ello, se empleó la ecuación:

$$
P R A=\frac{1}{r} \sum_{i=1}^{n}\left(\Delta M U_{i} R E i\right)+\left(\Delta M U_{i} R E e\right)+0.25\left[\left(\triangle A R F U R_{i} R E i\right)+\left(\triangle A R F U R_{i} R E e\right)\right]
$$

donde $P R A$ es la pérdida de renta agraria por urbanización, medida en \$c MM (millones de pesos constantes a diciembre de 2018); REi y REe es la renta económica de la tierra destinada, respectivamente, a producciones intensivas y extensivas; $\triangle M U$ y $\triangle A R F U R$ es la variación de superficie de la mancha urbana y el área de riesgo de la franja urbano-rural durante los años bajo estudio, medida en hectáreas; $r$ representa el costo de oportunidad de la tierra, donde se asume un valor del 5\%; y el subíndice $i$ de $1, \ldots, \mathrm{n}$ remite a las localidades estudiadas $(\mathrm{n}=69)$. El coeficiente 0,25 es un valor arbitrario, el cual representa el porcentaje del área de riesgo de la franja urbano-rural que se urbaniza y donde se observan múltiples usos no complementarios sobre este territorio.

La renta económica representa la diferencia entre el valor de lo producido y lo insumido en las producciones. La $R E e\left(\$ \mathrm{c} 13.435 \mathrm{ha}^{-1}\right)$ y $R E i\left(\$ \mathrm{c} 425.085 \mathrm{ha}^{-1}\right)$ se calculó a partir de datos locales (superficie y rendimiento), siguiendo el modo de 
cálculo de renta de Iglesias et al. (2017). Los precios corrientes se pasan a moneda constante (\$c) diciembre 2018 con el Índice de Precios Mayoristas Nivel General del INDEC. Para ello se utilizaron las series de precios de maíz (Zea mays), soja (Glycine max) y trigo (Triticum aestivum): diciembre 2015 a diciembre 2018 para la REe y la serie de precios de tomate (Lycopersicon esculentum) y lechuga (Latuca sativa) del año 2018 para la $R E i$. A los fines del cálculo de renta, se utilizaron los siguientes porcentajes de ocupación de los cultivos por hectárea: $15 \%$ y $85 \%$ para tomate y lechuga, respectivamente; $28,7 \%$ para maíz; $60,6 \%$ para soja y $10,7 \%$ para trigo.

\section{Expansión urbana y seguridad alimentaria}

La expansión urbana dispersa presenta un riesgo directo para la provisión de alimentos de proximidad. La desintegración espacial y funcional de la franja urbanorural genera pérdida de áreas productivas de alimentos de proximidad. La agricultura de proximidad se define como aquella actividad agropecuaria que produce alimentos con alta relación volumen-precio, que se caracteriza por el uso intensivo de recursos productivos y se realiza en cercanías urbanas, por cuestiones de disponibilidad de estos recursos y distancias para el traslado de la producción (Ermini et al., 2016; Feito et al., 2019). En este trabajo, los riesgos de la agricultura de proximidad se cuantifican mediante la producción y consumo aparente de alimentos frutihortícolas ( $\mathrm{AFH}$ ). El área de producción de estos alimentos se estimó a partir de censos agropecuarios (INDEC, 2002, 2008) e información publicada (Benencia et al., 2016). El consumo aparente se estimó con el valor de $400 \mathrm{gr} \mathrm{día}^{-1}$ por habitante de frutas y hortalizas no feculentas recomendado por la Organización Mundial de la Salud (oms, 2003). El consumo aparente se transforma en superficie equivalente con la productividad promedio de 21,1 toneladas por hectárea de cultivos frutihortícolas no feculentos publicados (FAOstaT: base de datos estadísticos corporativos de la Organización de las Naciones Unidas para la Alimentación y la Agricultura, FAO, 2018).

\section{Resultados}

\section{Evolución de la expansión urbana}

En los últimos 17 años, la tasa de crecimiento de las manchas urbanas ha triplicado la tasa de crecimiento de la población urbana en el sur de Córdoba (Tabla 1). Específicamente, la población urbana se incrementó 22\% (tasa anual de crecimiento $1,2 \%$ ), mientras que las manchas urbanas aumentaron en $86 \%$ (tasa anual de crecimiento 3,4\% y 4,1\% entre los ańos 2001-2010 y 2010-2018, respectivamente). Se urbanizaron 11.055 hectáreas y, consecuentemente, el área afectada por la expansión urbana ascendió a 39.648 hectáreas.

La evolución y dinámica de afectación del poblamiento urbano ha sido mayor en el segundo periodo de estudio. Durante el periodo 2001-2010, el crecimiento anual del área afectada por expansión urbana, mancha urbana y el área de riesgo de la franja urbano-rural fue en promedio 3,3\%, 3,4\% y 3,2\%, respectivamente (Tabla 1). En tanto, estos indicadores crecieron a un ritmo anual mayor en el período 2010-2018, en $5,9 \%$, 4,1\% y 9,3\%, respectivamente. De hecho, la magnitud de cambio de la tasa de crecimiento del perímetro de la mancha urbana (pasó de 1,9\% a 4,4\%) y 
la disminución en la densidad poblacional en la región (pasó de 25 a 16 hab. ha-1) muestran la agudización del fenómeno de expansión urbana.

TABLa I | Evolución del poblamiento urbano en el sur de Córdoba, Argentina

\begin{tabular}{|l|c|c|c|c|c|}
\hline \multirow{2}{*}{ AÑ̃O } & $\begin{array}{c}\text { POBLACIÓN } \\
\text { URBANA }\end{array}$ & AAEU & $\begin{array}{c}\text { MANCHA } \\
\text { URBANA }\end{array}$ & ARFUR & PERÍMETRO* $^{*}$ \\
\cline { 2 - 6 } & HAB. & HA & HA & HA & KM \\
\hline 2001 & $324.072 \mathrm{a}$ & 18.694 & 12.852 & 5.842 & 401 \\
\hline 2010 & $359.272 \mathrm{~b}$ & 25.133 & 17.381 & 7.752 & 475 \\
\hline 2018 & 394.462 & 39.648 & 23.907 & 15.741 & 668 \\
\hline TC 2001-2010 & $1,2 \%$ & $3,3 \%$ & $3,4 \%$ & $3,2 \%$ & $1,9 \%$ \\
\hline TC 2010-2018 & $1,2 \% * *$ & $5,9 \%$ & $4,1 \%$ & $9,3 \%$ & $4,4 \%$ \\
\hline
\end{tabular}

NOTA: AAEU (ÁREA AFECTADA POR EXPANSIÓN URBANA); * PERÍMETRO (PERÍMETRO DE LA MANCHA URBANA); ARFUR (ÁREA DE RIESGO DE LA FRANJA URBANO-RURAL); TC 20OI-20IO Y TC 2OIO20 I 8 (TASA ANUAL DE CRECIMIENTO PERIODO 2OOI-2OIO Y 2OIO-2OI8, RESPECTIVAMENTE);

** ESTIMADO SOBRE LA BASE DE LOS DATOS 2OOI-2OIO

FUENTE: ${ }^{A},{ }^{\text {B }}$ HTTP://WWW.CITYPOPULATION.DE/PHP/ARGENTINA-CORDOBA.PHP Y ELABORACIÓN PROPIA

FIGURA 3 | Evolución del área de riesgo de la franja urbano-rural en cuatro localidades del sur de Córdoba

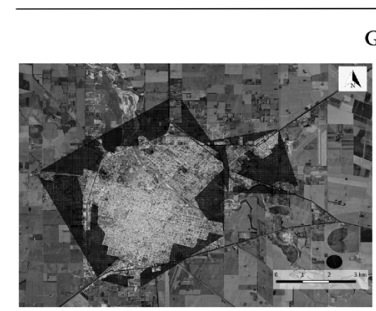

Año 2001: 2877 ha

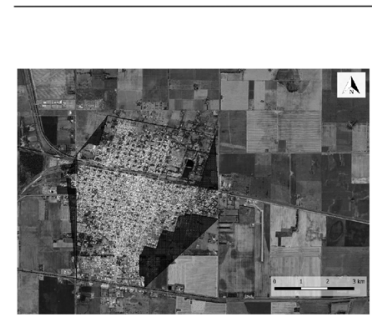

Año 2001: 159 ha

Gran Río Cuarto* $\quad 33^{\circ} 07^{\prime} 26^{\prime \prime S} ; 64^{\circ} 20^{\prime} 56^{\prime \prime} \mathrm{W}$

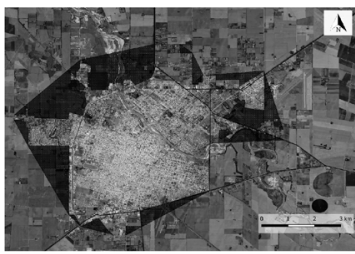

Año 2010: 3685 ha

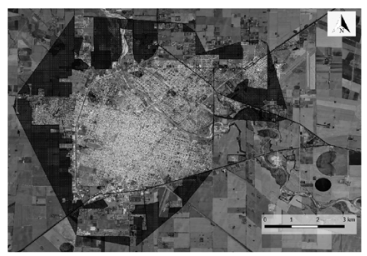

Año 2018: 4458 ha

Laboulaye $\quad 34^{\circ} 07^{\prime} 20^{\prime \prime} \mathrm{S} ; 63^{\circ} 23^{\prime} 15^{\prime \prime} \mathrm{W}$

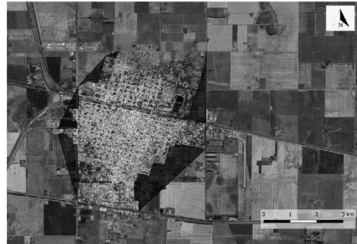

Ańo 2010: $198 \mathrm{ha}$

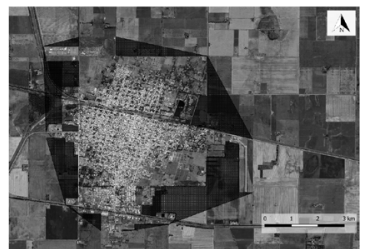

Ańo 2018: 549 ha

* integrado por localidades de río cuarto, Las higueras y santa catalina FUENTE: ELABORACIÓN PROPIA

Aunque el área de riesgo de la franja urbano-rural fue el indicador que mayor variabilidad y aumento registró entre periodos (Tabla 1), su evolución fue diferente 
según el tamaño de las localidades. En localidades de tamaño intermedio a grandes (> a 10.000 habitantes), esta área se amplió en promedio más del 160\% (Figura 3). En cambio, en localidades de menor tamaño ( $<1.250$ habitantes), el área de riesgo de la franja urbano-rural se redujo alrededor del 25\% y dichas localidades se están despoblando. En tanto, en localidades entre 10.000 y 1.250 habitantes, el área de riesgo de la franja urbano-rural muestra un comportamiento intermedio entre los extremos analizados.

\section{Poblamiento urbano}

El fenómeno de expansión urbana dispersa se multiplicó en la región. La cantidad de localidades que presentan poblamiento disperso, y su dinámica, varían de acuerdo al umbral de corte, indicador e índices probados. Por ello, es importante analizar los índices e indicadores en forma conjunta, para facilitar su interpretación y complementariedad.

FIGURA 4 | Poblamiento urbano por localidad, según la relación perímetro real y perímetro teórico de la mancha urbana (Ratio $P M U)$ e índice de expansión urbana dispersa (IEUD)

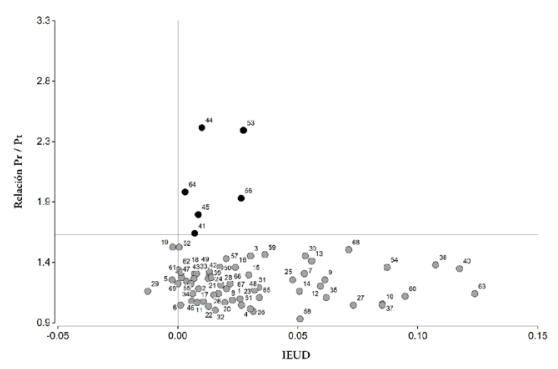

a) Periodo 2001-2010

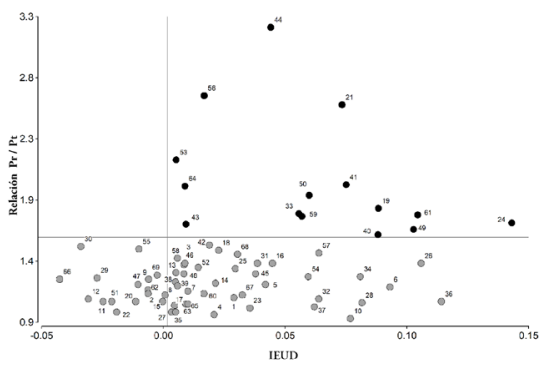

b) Periodo 2010-2018

NOTA: CÍRCULOS NEGROS Y GRIS: LOCALIDADES IDENTIFICADAS CON EL INDICADOR RATIO PMU E ÍNDICE IEUD RESPECTIVAMENTE (I: BUCHARDO; 2: DEL CAMPILLO; 3 : HUINCA RENANCÓ; 4 : ITALÓ; 5: JOVITA; 6: MATTALDI; 7: VILLA HUIDOBRO; 8: VILLA VALERIA; 9: NICOLÁS BRUZZONE; IO: ONAGOITY; II: PINCEN; I 2: RANQUELES; I 3: VILLA SARMIENTO; I 4: ALEJANDRO ROCA; I 5: BENGOLEA; I 6: CARNERILlO; I7: CHARRAS; I 8: GENERAL CABRERA; I 9: GENERAL DEHEZA; 20: HUANCHILLA; 2 I: LA CARLOTA; 22: LOS CISNES; 23: OLAETA; 24: REDUCCIÓN; 25: SANTA EUFEMIA; 26: UCACHA; 27: ASSUNTA; 28: EL RASTREADOR; 29: PACHECO DE MELO; 30 : PASO DEL DURAZNO; 3 I: GENERAL LEVALLE; 32: LA CESIRA; 33: LABOULAYE; 34: MELO; 35 : ROSALES; 36 : SERRANO; 37: VILLA ROSSI; 38: LEGUIZAMÓN; 39: RÍO BAMBA; 40: SAN JOAQUÍN; 4I: ACHIRAS; 42: ADELIA MARÍA; 43: ALCIRA GIGENA; 44: ALPA CORRAL; 45: BERROTARÁN; 46: BULNES; 47 : CHAJÁN; 48: CORONEL BAIGORRIA; 49: CORONEL MOLDES; 50 : ElENA; 5 I: LA CAUTIVA; 52 : LAS ACEQUIAS; 53: LAS HIGUERAS; 54: LAS VERTIENTES; 55: MONTE DE LOS GAUCHOS; 56: RÍO CUARTO; 57: SAMPACHO; 58: SAN BASILIO; 59: SANTA CATALINA; 60: TOSQUiTA; 6I: VICUÑa MACKeNNA; 62: CHUCUl; 63: LA CAROLINA "El pOTOSî"; 64: LAS ALbAHACAS; 65: LAS PEÑAS SUD; 66: MALENA; 67: SUCO; 68: VILLA EL CHACAY; 69: WASHINGTON)

FUENTE: ELABORACIÓN PROPIA

En la Figura 4 (a y b) se observa, para cada periodo de estudio, el tipo de poblamiento por localidad según IEUd y la relación de perímetros de la mancha urbana, Ratio PMU. El IEUd muestra dispersas a la mayoría de las localidades de la región. 
Respectivamente, durante el primer y segundo periodo, el 92\% y 77\% del total de las localidades presentaron este tipo de poblamiento. En tanto, el indicador Ratio $P M U$ indicó $9 \%$ de localidades con poblamiento disperso durante el primer periodo y $22 \%$ de localidades dispersas en el segundo periodo.

Estas diferencias son relativas al umbral de corte establecido para el indicador Ratio $P M U$. De hecho, cuanto más se aproxima a uno el valor del umbral de corte $(\alpha)$, la cantidad de localidades dispersas aumenta considerablemente. Por ello, es importante notar el resultado por localidad y la complementariedad entre este indicador y el IEUD, para identificar con precisión el fenómeno de expansión urbana en la región. De este modo, si observamos en el cuadrante superior derecho de la Figura 4 ( $\mathrm{a}$ y b) el número de localidades dispersas clasificadas por ambos índices entre periodos observados, pasa de 6 a 15 localidades dispersas.

\section{FIGURA 5 | Índice de compacidad por localidad según la relación perímetro real y perímetro teórico de la mancha urbana (Ratio PMU)}

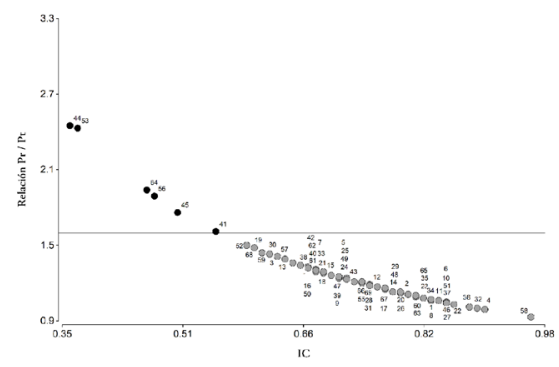

a) Periodo 2001-2010

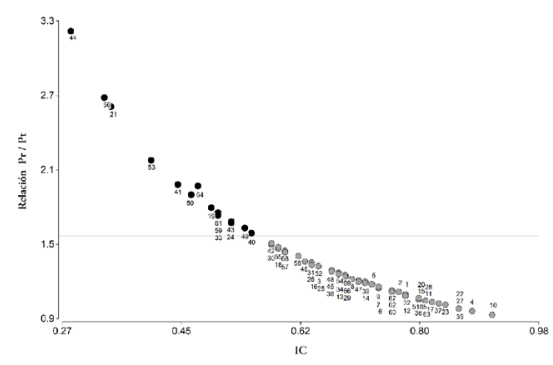

b) Periodo 2010-2018

NOTA: CÍRCULOS NEGROS Y GRIS: POBLAMIENTO URBANO IDENTIFICADO CON RATIO PMU E IEUD RESPECTIVAMENTE (I: BUCHARDO; 2: DEL CAMPILlO; 3: HUINCA RENANCÓ; 4: ITALÓ; 5: JOVITA; 6: MATTALDI; 7: VILLA HUIDOBRO; 8: VILlA VALERIA; 9: NICOlÁS BRUZZONE; IO: ONAGOITY; II: PINCEN; I 2: RANQUELES; I 3: VILLA SARMIENTO; I 4: ALEJANDRO ROCA; I 5: BENGOLEA; I 6: CARNERILLO; I7: CHARRAS; I 8: GENERAL CABRERA; I 9: GENERAL DEHEZA; 20: HUANCHILlA; 2 I: LA CARLOTA; 22: LOS CISNES; 23: OlAETA; 24: REDUCCIÓN; 25: SANTA EUFEMIA; 26: UCACHA; 27: ASSUNTA; 28: El RASTREADOR; 29: PACHECO DE MELO; 30 : PASO DEL DURAZNO; 3 I: GENERAL LEVALLE; 32: LA CESIRA; 33: LABOULAYE; 34: MELO; 35 : ROSALES; 36 : SERRANO; 37: VILLA ROSSI; 38: LEGUIZAMÓN; 39: RÍO BAMBA; 40: SAN JOAQUÍN; 4I: ACHIRAS; 42: ADELIA MARÍA; 43: ALCIRA GIGENA; 44: ALPA CORRAL; 45: BERROTARÁN; 46: BULNES; 47: CHAJÁN; 48: CORONEL BAIGORRIA; 49: CORONEL MOldeS; 50: ElENA; 5 I: LA CAUTIVA; 52 : LAS ACEQUIAS; 53: LAS HIGUERAS; 54: LAS VERTIENTES; 55: MONTE DE LOS GAUCHOS; 56: RÍO CUARTO; 57: SAMPACHO; 58: SAN BASILIO; 59: SANTA CATALINA; 60: TOSQUiTA; 6I: VICUÑA MACKENNA; 62: CHUCUL; 63: LA CAROLINA "EL POTOSÍ"; 64: LAS ALBAHACAS; 65: LAS PEÑAS SUD; 66: MALENA; 67: SUCO; 68: VILLA EL CHACAY; 69: WASHINGTON)

FUENTE: ELABORACIÓN PROPIA

Por otra parte, la evolución del índice de compacidad (IC) por localidad también remarcó el fenómeno de dispersión urbana (Figura 5). En el periodo 2001-2010, el valor promedio de IC de las localidades dispersas identificadas con el indicador Ratio PMU y, por ende, el IEUd, fue igual a $0,46(\sigma 0,08)$ y $0,45(\sigma 0,09)$ para el lapso 2010-2018. De hecho, en este último periodo se duplican las localidades dispersas con valores de IC menor a 0,45. Esta menor compacidad identificada en el segundo 
periodo de estudio subraya la expansión urbana dispersa, fenómeno que no puede ser capturado si solo se usa el IEUd. Por lo tanto, la inclusión del Ratio PMU complementa el IEUD y le otorga mayor precisión a la identificación del poblamiento.

Este hallazgo es muy importante para identificar tanto el patrón de poblamiento como los posibles conflictos urbano-rurales. El indicador Ratio PMU permite cuantificar la zona de contacto entre estos territorios, orientar sobre los riesgos asociados y las zonas más afectadas. Por ejemplo, si observamos en la Figura 4 la localidad de Río Cuarto, vemos que la ciudad pasa de un Ratio PMU de 1,9 a casi 2,7, incrementándose más del $80 \%$ dicha relación. Este indicador es independiente de la densidad de población y, por lo tanto, solamente captura la forma de expansión urbana dispersa sobre el medio rural.

\section{Expansión urbana y seguridad alimentaria}

Aunque el consumo de AFH aumentó en la región, la dinámica de la expansión urbana redujo significativamente la provisión de estos alimentos en la franja urbanorural del sur de Córdoba (Tabla 2). El consumo aparente de AFH (en t año-1) se incrementó en la misma medida que el crecimiento de población urbana, 22\%. En equivalente hectáreas, los requerimientos de áreas de producción para abastecer este consumo aparente crecieron de 2.240 hectáreas en el ańo 2001 a 2.726 hectáreas en el año 2018. Sin embargo, el área destinada a la producción de estos alimentos se redujo más del 50\% durante los ańos 2001-2018 (pasó de 795 a 333 hectáreas). Estas cifras ponen de manifiesto la creciente dependencia de AFH de fuentes externas a la región. En el año 2001, el área de producción representaba 35\% del área equivalente para abastecer el consumo aparente; y en el año 2018, esta proporción se redujo a $12 \%$.

TABLA 2 Área de producción y consumo aparente de alimentos frutihortícolas en el sur de Córdoba, Argentina

\begin{tabular}{|c|c|c|c|c|}
\hline \multirow{2}{*}{ AÑo } & \multicolumn{2}{|c|}{ CONSUMO APARENTE } & $\begin{array}{c}\text { ÁREA } \\
\text { PRODUCCIÓN }\end{array}$ & \multirow{2}{*}{ RELACIÓN P/C } \\
\cline { 2 - 4 } & T AÑO-I & EQUIVALENTE HA & HA & \\
\hline 2001 & 47.315 & 2.240 & 795 & 0,35 \\
\hline 2010 & 52.454 & 2.483 & 501 & 0,20 \\
\hline 2018 & 57.591 & 2.726 & 333 & 0,12 \\
\hline
\end{tabular}

NOTA: P/C = RELACIÓN PRODUCCIÓN (HA)/CONSUMO (EQUIVALENTE HA)

FUENTE: ELABORACIÓN PROPIA

Este hallazgo es relevante, ya que pone en evidencia la desintegración de la franja urbano-rural y la pérdida de una de sus funciones, esto es, la provisión de alimentos de proximidad. Desde el punto de vista económico, el ingreso de AFH desde otras regiones del país genera mayores costos de logística, y muy probablemente esta situación eleve los precios de dichos productos. Desde la perspectiva social, tanto los productores como los pobladores urbanos se ven perjudicados. La pérdida de los 
sistemas de producción AFH reduce las posibilidades de puestos de trabajo en proximidades urbanas y los consumidores disponen de una menor oferta de AFH locales. El incremento de la población urbana dispersa y de las zonas de contacto urbanorural acrecientan los riesgos de conflictos sociales por contaminación con agroquímicos, polución u olores fétidos. Desde una dimensión ambiental, el aumento en el transporte de AFH genera mayores emisiones de gases de efecto invernadero, mientras el desplazamiento de sistemas productivos hacia zonas alejadas de la mancha urbana genera áreas vacías y tierras en especulación en la interfase urbano-rural, las que suelen ser usadas para basurales a cielo abierto e incrementan el riesgo sanitario de la población.

\section{Expansión urbana y renta agraria}

En términos económicos, la evolución de la expansión urbana generó importantes transformaciones en la región. La conversión de 11.055 hectáreas de tierras rurales a áreas urbanizadas representó una pérdida de más del $70 \%$ del valor actual de la renta a perpetuidad de estas tierras; como consecuencia, los valores se aproximaron a \$c 43.912 мм у \$c 74.753 Mм para los periodos 2001-2010 y 2010-2018, respectivamente.

Hasta el momento, los hallazgos encontrados alertan sobre el fenómeno de expansión urbana dispersa en el sur de Córdoba, y los aspectos discutidos sitúan los valores analizados en contexto. Reconocida la dinámica de la dispersión urbana, es importante dilucidar y comparar las implicancias que tal dinámica tendrá a futuro en dos escenarios distintos: que se mantengan o se cambien las políticas y formas de poblamiento presentes. A esto nos abocamos en el próximo apartado.

\section{Prognosis de expansión urbana y proyecciones de poblamiento urbano}

Las brechas entre la prognosis y los patrones de poblamiento proyectados son significativas, aun considerando la misma población para el año 2040, estimada en 515.466 habitantes (Tabla 3). El área afectada por la expansión urbana quintuplicará el valor del año 2018, ascendiendo a 202.102 ha, mientras que los patrones alternativos compacto 1 y 2 afectarían solo 44.488 o 41.262 ha respectivamente. La mancha urbana de las 69 localidades se incrementa en $383 \%$ en la prognosis (91.679 ha) y se estima una pérdida de renta agraria por las tierras rurales afectadas de casi \$c 801 mM. En tanto, los patrones de poblamiento alternativos usarían solo alrededor del $30 \%$ de la tierra urbanizada en la prognosis (28.747 ha en la forma Compacta-1 y 25.521 ha en la forma Compacta-2). En consecuencia, el área afectada por urbanización y el perímetro de contacto urbano-rural, y la pérdida de renta agraria, podrían reducirse en promedio 87\% y 67\%, y en más de \$c 650 MM, respectivamente. 
TABLA 3 | Poblamiento urbano año 2040 en el sur de Córdoba

\begin{tabular}{|c|c|c|c|c|c|}
\hline \multirow{2}{*}{ PATRÓN } & $\begin{array}{c}\text { POBLACIÓN } \\
\text { URBANA }\end{array}$ & AAEU & $\begin{array}{c}\text { MANCHA } \\
\text { URBANA }\end{array}$ & PERÍMETRO* & PRA \\
\cline { 2 - 6 } & HAB. & HA & HA & KM & MM \$C \\
\hline Prognosis** & 515.466 & 202.102 & 91.679 & 2.532 & 801.986 \\
\hline Compacto-1 & 515.466 & 44.488 & 28.747 & 807 & 161.115 \\
\hline Compacto-2 & 515.466 & 41.262 & 25.521 & 748 & 132.815 \\
\hline
\end{tabular}

NOTA: AAEU (ÁREA AFECTADA POR EXPANSIÓN URBANA); *PERÍMETRO (PERÍMETRO DE LA MANCha

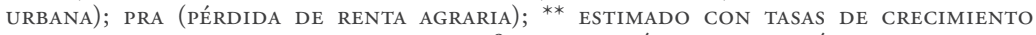
CORRESPONDIENTES AL PERIODO 2OIO-2OI8, A EXCEPCIÓN DE POBLACIÓN URBANA DONDE SE UTILIZA LA TASA INTERCENSAL (AÑOS 200I-20IO). EL VALOR ACUMULADO EN LA FILA PROGNOSIS SE REALIZA POR LOCALIDAD Y LUEGO LA SUMATORIA DE LOS 69 CASOS BAJO ESTUDIO

FUENTE: ELABORACIÓN PROPIA

Las modalidades de poblamiento más compactas orientarían a los gobiernos municipales y provinciales, como también nacionales, a inducir comportamientos sociales más sostenibles en el territorio. Los patrones compactos de expansión urbana futura minimizan la conversión de tierras rurales y los esfuerzos de gestión para el desarrollo de infraestructuras y servicios públicos alejados de la mancha urbana. Desde lo económico, se resguarda la renta agraria y se reducen los costos de inversión y mantenimiento de las infraestructuras públicas. Además, si se jerarquizan las funciones de la franja urbana -tales como generación de alimentos de proximidad bajo diferentes formas de producción, aprovechamiento de efluentes y gestión de residuos sólidos urbanos y desarrollo de zonas de amortiguación y cercos verdes para facilitar el uso mixto del suelo-, muy probablemente los gobiernos y actores involucrados puedan alinearse hacia un modelo de desarrollo económico y territorial de largo plazo más sostenible para el sur de Córdoba.

\section{Discusión}

En primer lugar, los valores de conversión anual de tierras rurales a urbanas de 3,4\% y 4,5\% hallados para los ańos 2001-2010 y 2010-2018 respectivamente (Tabla 1), son semejantes a valores encontrados en la bibliografía. Por ejemplo, Kasanko et al. (2006), en quince ciudades europeas, encontraron un valor de conversión anual de tierras rurales de 3,5\% entre las décadas de 1950 y 1970 . Del mismo modo, pero en New Jersey, Hasse y Lathrop (2003) describieron un crecimiento anual del área urbana mayor al 5,5\% entre los años 1986 y 1995. Más recientemente, Angel et al. (2011), en un análisis global sobre 200 ciudades, mencionaron una conversión anual de tierras rurales del 1,9\% entre los ańos 1990 y 2015, con tendencia al aumento, que aparece más alarmante para países en desarrollo. En tanto, en Argentina también se describen valores semejantes de tasas de conversión, como 3,3\% en Mar del Plata entre los ańos 2003 y 2009 (Zulaica \& Ferraro, 2013); 3,5\% 
en Córdoba entre los años 2014 y 2016 (Gordillo \& Giobellina, 2017); y 3\% en Río Cuarto entre los años 1992 y 2002 (Maldonado \& Campanella, 2004).

En segundo lugar, los valores de conversión anual de tierras rurales superan la tasa de crecimiento anual de población urbana. En el área de estudio, esta situación se encuentra entre 2 a 3 puntos de diferencia según el periodo observado (Tabla 1) y entre 2 a 7 puntos del rango hallado por otros autores (Angel et al., 2011; Kasanko et al., 2006).

En tercer lugar, aunque el número de localidades dispersas identificadas en el año 2018 es semejante al de localidades "periurbanizadas" indicadas por Margonari y Menendez (2019), los resultados difieren sensiblemente. En 2019, estos autores muestran en su contribución un mapa de la provincia de Córdoba, donde 12 localidades de la región sur presentan procesos de "periurbanización". Sin embargo, en este trabajo identificamos, para el año 2018, 15 localidades dispersas con el indicador Ratio PMU y 53 localidades con el IEUd. Particularmente, 7 de las 15 localidades indicadas por el Ratio PMU (Achiras, Alpa Corral, Albahacas, Reducción, Elena, Santa Catalina y San Joaquín) difieren de las señaladas por estos autores. Posiblemente, esta diferencia observada se deba al tipo de metodología utilizada en el sistema de información geográfico para la identificación del poblamiento urbano (p.e., el proceso de digitalización de las manchas urbanas con mayores detalles).

\section{Conclusiones}

Este artículo analiza la evolución y los riesgos de la expansión urbana en 69 localidades del sur de Córdoba, Argentina, durante los periodos 2001-2010 y 20102018. En primer lugar, se identifica la evolución del poblamiento urbano como disperso o no disperso mediante un índice de expansión urbana dispersa (IEUD), complementado a un indicador dispuesto para este trabajo, Ratio PMU o relación de perímetros de las manchas urbanas y un índice de compacidad (IC). En segundo lugar, se describen los riesgos de la dispersión urbana sobre la provisión de alimentos de proximidad y se valora la pérdida económica de las tierras rurales que se urbanizan. Finalmente, se exploran las implicancias del poblamiento actual frente a dos patrones alternativos al año 2040 .

Un hallazgo relevante es el incremento de la dispersión urbana en la región. Los índices usados para identificar el patrón de poblamiento urbano reflejan esta situación y, particularmente, el Ratio PMU muestra el doble de localidades dispersas entre periodos de estudio. Este indicador captura las relaciones de contacto urbano-rural con el perímetro de la mancha urbana y permite cuantificar las posibles áreas de conflicto entre estos territorios. De hecho, en la región pampeana del país, el riesgo por contaminación con agroquímicos en las zonas de contacto urbano-rural es el principal problema ambiental percibido por la población urbana.

Este aumento del poblamiento urbano disperso presenta diferentes implicancias y riesgos para los gobiernos locales, la comunidad y el desarrollo territorial. La urbanización de tierras rurales se ha duplicado, para albergar menos de un cuarto del crecimiento poblacional, y las áreas de producción de alimentos de proximidad se redujeron más de la mitad. Esta situación resulta en mayores esfuerzos políticos y 
presupuestarios para mejorar los equipamientos urbanos y la provisión de servicios básicos a la población. También se redujo significativamente el abastecimiento local de alimentos de proximidad. Esto aumenta la desintegración espacial y funcional de la franja urbano-rural.

Si se mantienen las mismas políticas de poblamiento a futuro, estos efectos indeseados se agravarán significativamente; sin embargo, existen al menos dos formas de urbanización que identifican una visión futura socialmente más deseable. Mantener las actuales políticas de poblamiento para el año 2040 significa un aumento alrededor tres veces mayor de las manchas urbanas para albergar solo $37 \%$ de la población adicional. En contraste, formas de poblamiento más compactas menguan las implicancias antes descriptas. La urbanización de tierras rurales, la pérdida de renta económica asociada y las zonas de contacto urbano-rural pueden minimizarse o estar en orden de magnitud al adicional de población esperada. Particularmente, esta brecha entre la prognosis y los modelos alternativos de poblamiento urbano deberían orientar a los gobiernos locales sobre la posibilidad de inducir comportamientos sociales más sostenibles para el desarrollo territorial.

Aunque los resultados hallados son consistentes, el lector debe considerar ciertas limitaciones. En primer lugar, el cálculo del consumo aparente de los alimentos de proximidad y la valoración de la productividad de las tierras frutihortícolas se realiza con valores constantes (de la OMS y FAO), sin precisión. En segundo lugar, la estimación de los patrones de poblamiento alternativos (compacto 1;2) se tomaron de parámetros de la bibliografía sin considerar los ajustes por cada localidad. Por ello, los resultados obtenidos tienen validez relativa en el contexto del trabajo y no en forma absoluta. En tercer lugar, el crecimiento de población urbana se realizó a través de un modelo geométrico con datos censales (2001 y 2010) que no incluyen valores de natalidad, mortalidad y migración. Por último, la figura geométrica elegida para conceptualizar el perímetro teórico de la mancha urbana para estimar el Ratio $P M U$ es válida para las morfologías urbanas de la región pampeana y su extrapolación a otras regiones debe ser ajustada a figuras que mejor representen la morfología. Estas limitaciones son parte de la agenda futura de investigación.

\section{Referencias bibliográficas}

Ackoff, R. (1994). Systems thinking and thinking systems. System Dynamics Review, 10, $175-$ 188. https://doi.org/10.1002/sdr.4260100206

Alberti, M. (2005). The effects of urban patterns on ecosystem function. International Regional Science Review, 28(2), 168-192. https://doi.org/10.1177/0160017605275160

Angel, S., Parent, J. \& Civco, D. (2012). The fragmentation of urban landscapes: Global evidence of a key attribute of the spatial structure of cities, 1990-2000. Environment and Urbanization 24, 249-283. https://doi.org/10.1177/0956247811433536

Angel, S., Parent, J., Civco, D. L., Blei, A. \& Potere, D. (2011). The dimensions of global urban expansion: Estimates and projections for all countries, 2000-2050. Progress in Planning, 75(2), 53-107. https://doi.org/10.1016/j.progress.2011.04.001 
ваотсbа (Bases ambientales para el ordenamiento territorial del espacio rural de la provincia de Córdoba). (2010). Bases ambientales para el ordenamiento territorial del espacio rural de la provincia de Córdoba. Universidad Nacional de Córdoba (Secretaría de Ciencia y Tecnología) y Universidad Nacional de Río Cuarto. http://www. ordenamientoterritorialcba.com/web3/

Barsky, A. (2005). El periurbano productivo, un espacio en constante transformación. Introducción al estado del debate, con referencias al caso de Buenos Aires. Scripta Nova. Revista Electrónica de Geografía y Ciencias Sociales, 9(36). http://www.ub.es/ geocrit/sn/sn-194-36.htm

Benencia, R., Salusso, F. \& Ramos, D. (2016). Inserción de horticultores bolivianos en Río Cuarto. Procesos de inmigración, trabajo y conformación de economías étnicas. Mundo Agrario, 17(36), e029. https://www.mundoagrario.unlp.edu.ar/article/view/ MAe029

Bertoni, J. C. (2006). Inondations urbaines en Amérique Latine: réflexions sur le rôle des facteurs de risque. Frontiers in Flood Research, 305, 123-141. https://iahs.info/uploads/ dms/13518.10-123-142-305-08-Bertoni.pdf

Boccolini, S. M. \& Giobellina, B. (2018). Reconstrucción histórica del territorio periurbano de producción hortícola de Córdoba, Argentina (1573-1900). Eutopía. Revista De Desarrollo Económico Territorial, (14), 83-110. https://doi.org/10.17141/ eutopia.14.2018.3577

Burton, E. (2000). The compact city: Just or just compact? A preliminary analysis. Urban Studies, 37(11), 1969-2001. https://doi.org/10.1080/00420980050162184

Burton, E., Jenks, M. \& Williams, K. (Eds.). (2003). The compact city: a sustainable urban form? Routledge. https://doi.org/10.4324/9780203362372

Cabrini, S., Cristeche, E., de Prada, J., Dupleich, J., Engler, P., Espósito, M., Manchado, J. C., Mathey, D., Natinzon, P., Schutz, P., Tello, D. \& Vicente, G. (2018). Percepción sobre el impacto ambiental de la producción agropecuaria de la región pampeana argentina. En F. M. Suárez \& C. A. Ruggerio (Comps.), Los conflictos ambientales en América Latina I: Casos y reflexiones (pp. 387-410). Ediciones Universidad Nacional General Sarmiento. Colección Ambiente y Territorio. https://inta.gob.ar/sites/default/files/ inta_cicpes_instdeeconomia_cristeche_percepcion_sobre_el_impacto.pdf / https:// ediciones.ungs.edu.ar/wp-content/uploads/2018/10/9789876303668-completo.pdf

Carruthers, J. I. \& Ulfarsson, G. F. (2003). Urban sprawl and the cost of public services. Environment and Planning B: Planning and Design, 30, 503-522. https:/doi. org/10.1068/b12847

de Prada, J., Degioanni, A., Cantero, A., Tello, D., Gil, H., Cahe, E., Cisneros, J., Becerra, V. \& Pereyra, C. (2017a). Procedimiento multicriterio en fases para la construcción de la visión territorial local. Aplicación en la localidad de Santa Eufemia, Córdoba, Argentina. Revista Argentina de Economía Agraria, 17(1), 6-30. https://raea.com.ar/ revistaaaea_arg/article/view/23

de Prada, J., Degioanni, A., Cisneros, J. M., Cantero, A., Gil, H., Tello, D., Pereyra, C. \& Giayetto, O. (2017b). Planificación del territorio: Elección del patrón de urbanización. El caso de la ciudad de Río Cuarto, Córdoba, Argentina. Revista de Métodos Cuantitativos para la Economía y la Empresa, 26, 25-51. https://www.upo.es/ revistas/index.php/RevMetCuant/article/view/2630 
Dodman, D. (2009). Urban density and climate change. En United Nations Population Fund (UNFPA), Analytical review of the Interaction between Urban Growth Trends and Environmental Changes (Paper 1). https://www.uncclearn.org/wp-content/uploads/ library/unfpa14.pdf

Ermini, P., Giobellina, B. \& Barsky, A. (2016). Caracterización de la agricultura de proximidad al área metropolitana de Santa Rosa-Toay (La Pampa, Argentina): aportes para la discusión sobre soberanía alimentaria. Huellas, (20), 125-143. http://dx.doi. org/10.19137/huellas-2016-2007

Feito, M. C., Boza, S. \& Peredo, S. (2019). La agricultura en los periurbanos de Buenos Aires (Argentina) y Santiago (Chile): Territorios en transición. Revista del Area de Estudio Urbanos del Instituto de Investigaciones Gino Germani de la Facultad de Ciencias Sociales (UBA), 16(11), 32-54. https://publicaciones.sociales.uba.ar/index.php/quid16/article/ view/3138/pdf

Food and Agriculture Organization of the United Nations (ғаолтAт). Database. (2018). Crops Production and Livestock Primary. http://www.fao.org/faostat/en/\#data/QC

Gaffron, P., Huismans, G. \& Skala, F. (Coords.). (2008). Proyecto Ecocity. Manual para el diseño de ecociudades en Europa. Libro 2: La ecociudad: cómo hacerla realidad. Gea21 / Bakeaz / Ministerio de Vivienda, Gobierno de España. http://www.estudislocals.cat/wp-content/ uploads/2016/11/proyecto_ecocity_la_ecociudad_como_hacerla_realidad.pdf

Galfioni, M., Degioanni, A., Maldonado, G. \& Campanella, O. (2013). Conflictos socioambientales: identificación y representación espacial. Estudio de caso en la ciudad de Río Cuarto (Argentina). Estudios Geográficos, 74(275), 469-493. https:// doi.org/10.3989/estgeogr.201317

Gallent, N. (2006). The Rural-Urban fringe: A new priority for planning policy? Planning Practice \& Research, 21(3), 383-393. https://doi.org/10.1080/02697450601090872

Gallent, N. \& Shaw, D. (2007). Spatial planning, area action plans and the rural-urban fringe. Environmental Planning and Management, 50(5), 617-638. https://doi. org/10.1080/09640560701475188

Gao, B., Huang, Q., He, C., Sun, Z. \& Zhang, D. (2016). How does sprawl differ across cities in China? A multi-scale investigation using nighttime light and census data. Landscape and Urban Planning, 148, 89-98. https://doi.org/10.1016/j.landurbplan.2015.12.006

Gómez Orea, D. (2008). Ordenación territorial. 2a edición. Ediciones Mundi Prensa.

Gordillo, N. \& Giobellina, B. (2017). Transformaciones territoriales en la interfase urbanorural de Córdoba. La producción frutihortícola del Cinturón Verde de la ciudad de Córdoba, como servicio ecosistémico. X Seminario Internacional de Investigación en Urbanismo. Barcelona-Córdoba, junio 2018. Universidad Nacional de Córdoba / Universitat Politècnica de Catalunya, Departament d'Urbanisme i Ordenació del Territori.

Hasse, J. \& Lathrop, R. (2003). Land resource impact indicators of urban sprawl. Applied Geography, 23(2), 159-175. https://doi.org/10.1016/j.apgeog.2003.08.002

Hortas-Rico, M. (2014). Urban sprawl and municipal budgets in Spain: A dynamic panel data analysis. Barcelona Institute of Economics (IEB) and Department of Public Finance and Tax System, Complutense University of Madrid. Papers in Regional Science, 93(4), 843-865. https://doi.org/10.1111/pirs. 12022 
Huang, J., Lu, X. \& Sellers, J. (2007). A global comparative analysis of urban form: Applying spatial metrics and remote sensing. Landscape and Urban Planning, 82(4), 184-197. https://doi.org/10.1016/j.landurbplan.2007.02.010

Iglesias, D., Lorda, H., Porto, R. \& Fernández, M. (2017). INTA. Boletín Económico Nº 32, Redes de Economía Agropecuaria de La Pampa y San Luis. https://inta.gob.ar/sites/ default/files/inta_informe_marzo2017.pdf

Instituto Nacional de Estadística y Censos (INDEC). (2001). Censo Nacional de Población, Hogares $y$ Viviendas 2001. https://www.indec.gob.ar/indec/web/Nivel4-Tema-2-41-134

Instituto Nacional de Estadística y Censos (INDEC). (2002). Censo Nacional Agropecuario 2002. Córdoba. Hortalizas. eAp con límites definidos. Superficie implantada a campo y bajo cubierta, por especie, según departamento. https://sitioanterior.indec.gob.ar/ cna_index.asp

Instituto Nacional de Estadística y Censos (INDEC). (2008). Censo Nacional Agropecuario 2008. Córdoba. Hortalizas. EAP con limites definidos. Superficie implantada a campo y bajo cubierta, por especie, según departamento. https://sitioanterior.indec.gob.ar/nivel4_ default.asp?id_tema_1=38id_tema_2=8\&id_tema_3=87

Instituto Nacional de Estadística y Censos (INDEC). (2010). Censo Nacional de Población, Hogares y Viviendas 2010. https://www.indec.gob.ar/indec/web/Nivel4-Tema-2-41-135

Jabareen, Y. R. (2006). Sustainable urban forms: their typologies, models, and concepts. Journal of Planning Education and Research, 26(1), 38-52. https://doi. org/10.1177/0739456X05285119

Jenks, M. \& Dempsey, N. (2005). Future forms and design for sustainable cities. Architectural Press.

Kasanko, M., Barredo, J., Lavalle, C., McCormick, N., Demicheli, L., Sagris, V. \& Brezger, A. (2006). Are European cities becoming dispersed? A comparative analysis of 15 European urban areas. Landscape and Urban Planning, 77(1), 111-130. https://doi. org/10.1016/j.landurbplan.2005.02.003

Liao, Y. \& Chern, S. (2015). Strategic ecocity development in urban-rural fringes: Analyzing Wulai District. Sustainable Cities and Society, 19, 98-108. https://doi.org/10.1016/j. scs.2015.07.014

López Goyburu, P. (2016). Las cuencas y el periurbano. El caso de la cuenca del río MatanzaRiachuelo y la cuenca del río Reconquista. Ciencias del Agro, Ingeniería y Tecnología, 7(17), 45-57. https://produccioncientificaluz.org/index.php/rluz/article/view/30936

Lopez, R. (2014). Urban sprawl in the United States: 1970-2010. Cities and the Environment (CATE), 7(1), 7. http://digitalcommons.lmu.edu/cate/vol7/iss1/7

Maldonado, G. \& Campanella, O. (2004). Evolución de la mancha urbana de la ciudad de Río Cuarto, Córdoba, Argentina, mediante la aplicación de tecnología de sensoramiento remoto y sistemas de información geográfica. $\sigma^{\circ}$ Encuentro Internacional Humboldt. Villa Carlos Paz, Argentina.

Margonari, A. \& Menendez, A. (2019). ¿Qué entendemos por "periurbano"? [Contribución técnica al estudio territorial inmobiliario: interfaz rural-urbana]. Ministerio de Finanzas, Gobierno de Córdoba. https://idecor.cba.gov.ar/que-entendemos-porterritorio-periurbano/

Montero, M. (2011). Usos del suelo en la ciudad de Río Cuarto: sector sur. Huellas, 15, 166179. http://www.biblioteca.unlpam.edu.ar/pubpdf/huellas/v15a12montero 
Moore, N. (2016). Reseña: The Green City: Sustainable homes, sustainable suburbs, de N. Low, B. Gleeson, R. Green \& D. Radovic. Urban Geography, 30(8), 927-928. https://doi. org/10.2747/0272-3638.30.8.927

Munton, R. (2009). Rural land ownership in the United Kingdom: Changing patterns and future possibilities for land use. Land Use Policy, 26(1), 54-61. https://doi. org/10.1016/j.landusepol.2009.08.012

Neirotti, P., De Marco, A., Cagliano, A., Mangano, G. \& Scorrano, F. (2014). Current trends in Smart City initiatives: Some stylised facts. Cities, 38, 25-36. https://doi.org/10.1016/j. cities.2013.12.010

Organización Mundial de la Salud (oms). (2003). Dieta, nutrición y prevención de enfermedades crónicas. https://www.who.int/mediacentre/news/releases/2003/pr20/es/

Pauleit, S., Ennos, R. \& Golding, Y. (2005). Modeling the environmental impacts of urban land use and land cover change-a study in Merseyside, uk. Landscape and Urban Planning, 71(2), 295-310. https://doi.org/10.1016/j.landurbplan.2004.03.009

Puig, H. S. (2016). El periurbano, un espacio estratégico de oportunidad. Revista Bibliográfica de Geografía y Ciencias Sociales [Universidad de Barcelona], 21(1), 160. http://www. ub.edu/geocrit/b3w-1160.pdf

Samaniego, L. \& Bárdossy, A. (2006). Simulation of the impacts of land use/cover and climatic changes on the runoff characteristics at the mesoscale. Ecological Modelling, 196(1), 45-61. https://doi.org/10.1016/j.ecolmodel.2006.01.005

Schneider, A. \& Woodcock, C. E. (2008). Compact, dispersed, fragmented, extensive? A comparison of urban growth in twenty-five global cities using remotely sensed data, pattern metrics and census information. Urban Studies, 45(3), 659-692. https://doi. org/10.1177/0042098007087340

Schwarz, N. (2010). Urban form revisited-Selecting indicators for characterising European cities. Landscape and Urban Planning, 96(1), 29-47. https://doi.org/10.1016/j. landurbplan.2010.01.007

Scott, A. J., Carter, C., Reed, M. R., Larkham, P., Adams, D., Morton, N., Waters, R., Collier, D., Crean, C., Curzon, R., Forster, R., Gibbs, P., Grayso, N., Hardman, M., Hearle, A., Jarvis, D., Kennet, M., Leach, K., Middleton ... Coles, R. (2013). Disintegrated development at the rural-urban fringe: Re-connecting spatial planning theory and practice. Progress in Planning, 83, 1-52. https://doi.org/10.1016/j. progress.2012.09.001

Sereno, C. \& Serer Santarelli, S. A. (2012). El rururbano: un espacio de vulnerabilidad y riesgo. Estudio cualitativo en la ciudad de Bahía Blanca, provincia de Buenos Aires, Argentina. Revista Colombiana de Geografía, 21(2), 149-165. https://doi.org/10.15446/rcdg. v21n2.28263

Stone, B., Hess, J. \& Frumkin, H. (2010). Urban form and extreme heat events: Are sprawling cities more vulnerable to climate change than compact cities? Environmental Health Perspectives, 118(10), 1425-1428. https://doi.org/10.1289/ehp.0901879

Torres-Degró, A. (2017). Tasas de crecimiento poblacional (r): Una mirada desde el modelo matemático lineal, geométrico y exponencial. CIDE Digital, 2(1), 143-162. https:// revistas.upr.edu/index.php/cidedigital/article/view/11774 
Urriza, G. \& Garriz, E. (2014). ¿Expansión urbana o desarrollo compacto? Estado de situación en una ciudad intermedia: Bahía Blanca, Argentina. Revista Universitaria de Geografía, 23(2), 97-124. https://www.redalyc.org/articulo.oa?id=383239105003

Venier, G. (2012). Desarrollo territorial: abordaje de procesos complejos en la nueva ruralidad. Proyecto BUR - Bordes Urbanos Rurales. Construyendo Vinculos (Inta Ediciones), 2, 4762. https://inta.gob.ar/sites/default/files/inta.revista-construyendo-vinculos.pdf

Zulaica, L. \& Ferraro, R. (2013). Lineamientos para el ordenamiento del periurbano de la ciudad de Mar del Plata (Argentina), a partir de la definición de sistemas territoriales. Geografia em questao, 6(1), 202-230. http://e-revista.unioeste.br/index.php/geoemques tao/article/view/6731 